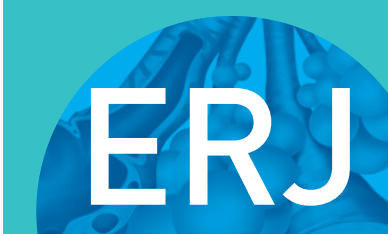

open research
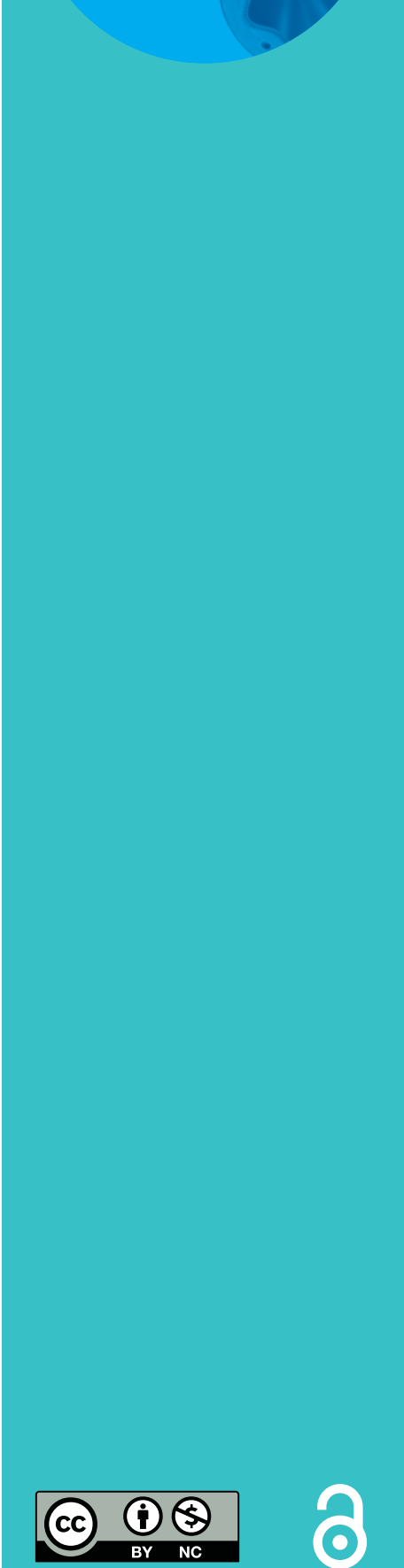

\section{Factors associated with delay in treatment initiation for pulmonary tuberculosis}

\author{
David J. Roberts (1) ${ }^{1,2,3}$, Trish Mannes ${ }^{3}$, Neville Q. Verlander ${ }^{4}$ and \\ Charlotte Anderson ${ }^{5}$
}

Affiliations: ${ }^{1}$ Field Epidemiology Training Programme, Public Health England Colindale, London, UK. ${ }^{2}$ European Programme for Interventional Epidemiology Training (EPIET), European Centers for Disease Control, Stockholm, Sweden. ${ }^{3}$ Thames Valley Health Protection Team, Public Health England South East, Chilton, UK. ${ }^{4}$ Statistics, Modelling and Economics Dept, Public Health England Colindale, London, UK. ${ }^{5}$ Public Health England, Field Service South East and London, London, UK.

Correspondence: David J. Roberts, Public Health England - Health Protection (South East Centre), PHE Chilton, Didcot, OX11 ORQ, UK. E-mail: david.j.robertsaphe.gov.uk

\section{ABSTRACT}

Background: Delays in treatment initiation for tuberculosis (TB) may lead to worse clinical outcomes and increased transmission. We aimed to determine factors associated with treatment delays, to guide public health action.

Methods: We extracted data on clinical characteristics and documented potential barriers to treatment from all pulmonary TB cases with clinical case review data from 2011 to 2015 and linked these to TB surveillance data. We described the distribution of delays from symptom onset to first presentation ("presentation delay") and from presentation to treatment ("healthcare delay"). We calculated time ratios (TRs) to determine the association between sociodemographic and clinical factors and delay outcomes.

Results: Median presentation delay was 30 days (interquartile range (IQR) 11-72 days). Language barriers were associated with $40 \%$ longer presentation delay (TR 1.40, 1.01-1.94). Median healthcare delay was 40 days (IQR 13-89 days), and mostly consisted of the time taken before deciding to refer to TB specialists (median 26 days, IQR 4-73 days). Shorter healthcare delay was associated with positive sputum smear (TR $0.58,0.47-0.70$ ), UK residency $<2$ years (TR 0.47, 0.32-0.67), male sex (TR 0.74, 0.60-0.91) and secondary care referral (TR 0.63, 0.51-0.78).

Conclusions: Our findings support continued initiatives to enable access to care for migrant populations to minimise presentation delay. Multifaceted approaches to increase clinician awareness of TB clinical presentations, to implement systems enabling early case recognition, to maximise the yield from sputum smear investigations and to ensure rapid diagnosis of smear negative cases are required to achieve further TB control.

@ERSpublications

What factors are associated with delayed pulmonary tuberculosis treatment initiation? Language barriers are associated with delayed presentation to healthcare. Smear negativity, female sex and longer residence are associated with delays after presentation. http://bit.ly/2MWRIHS

Cite this article as: Roberts DJ, Mannes T, Verlander NQ. Factors associated with delay in treatment initiation for pulmonary tuberculosis. ERJ Open Res 2019; 6: 00161-2019 [https://doi.org/10.1183/ 23120541.00161-2019].

This article has supplementary material available from openres.ersjournals.com.

Received: 17 Oct 2019 | Accepted: 17 Oct 2019

Copyright $\odot$ ERS 2020. This article is open access and distributed under the terms of the Creative Commons Attribution Licence 4.0. 


\section{Background}

Longer intervals from tuberculosis (TB) symptom onset to starting anti-TB chemotherapy contribute to adverse public health outcomes of increased transmission to contacts and adverse clinical outcomes for people with TB [1]. Despite recommendations that health services facilitate rapid referral to specialist TB services to confirm diagnosis and initiate treatment when $\mathrm{TB}$ is suspected [2], long delays between symptom onset and treatment are common in England: in 2017, 31\% of people with pulmonary TB (pTB) experienced a delay of $\geqslant 4$ months, and the median time between symptom onset and treatment start was 79 days [3]. In 2015, the South East (incidence 7/100000 cases per year), had the highest percentage nationally of people with pTB yet to start treatment by 2 months or 4 months after symptom onset [4]. Improving access to care and ensure early diagnosis is a key area for action defined in the national Collaborative Tuberculosis Strategy for England 2015-2020 [5].

Broadly, delays in treatment initiation occur between symptom onset and presentation ("presentation delay" (presentation delay)) or within the healthcare system between presentation and initiating treatment ("healthcare delay"). Enhanced TB Surveillance (ETS) data have been used to evaluate the factors associated with delays in England. However, ETS has important limitations in terms of data completeness for some variables, and comprehensiveness of coverage of data on potential risk factors for delay. By linking ETS data with an alternative data source to overcome these limitations, we aimed to identify where in the TB presentation and care pathway people face delays in the initiation of treatment, and which groups or areas are most affected, to target public health interventions and future research.

\section{Methods}

\section{Study design and subjects}

We conducted a retrospective cohort study. The study population included residents in the South East of England with pTB from 2011 to 2015 inclusive. Cases were defined as pTB if they had any evidence of pTB involvement.

\section{Data sources and data handling}

Cohort review is the systematic appraisal of the case management and contact investigation of every TB case [6], and was gradually rolled out across the South East from 2011. At cohort review, TB nurses retrospectively complete case-level forms, capturing sociodemographic, clinical, treatment and contact tracing information. We collated and harmonised cohort review data from all four regional Public Health England (PHE) Health Protection Teams (HPTs) to create a single regional dataset. Data for this study were extracted from both cohort review and ETS datasets. Where available for the same variable, cohort review data were used preferentially, as we considered that they were likely to be more complete and accurate than ETS data. We extracted data from both data sources, but mental health barriers, language barriers, chest radiography findings at diagnosis and referral source data were extracted from cohort review only. Data on Lower layer Super Output Area (LSOA) level index of multiple deprivation 2015 (Department for Communities and Local Government), LSOA-level urban rural classification from Office for National Statistics survey 2011 data, and local authority district (LAD) were linked using postcode of residence at notification. LAD TB incidence was classified based on the PHE 2016 annual TB report [4]. We included the following social risk factors: any history of drug misuse, homelessness and/or imprisonment. Additionally, current alcohol misuse, mental health barriers and language barriers were all defined by affecting the person's ability to self-medicate, as judged by the TB nurse completing the form. We extracted date of symptom onset, date first presented to health services, date of referral to TB services (cohort review only), date first seen by TB services (cohort review only), date of diagnosis and date of starting TB treatment. We generated outcome delay variables as the interval in days between the date of the earlier point in the clinical pathway from the later date (supplementary figure S1). The interval between symptom onset and presentation and between presentation and treatment initiation were defined as in the introduction. Healthcare delay was further subdivided into the interval between presentation and referral to TB services ("decision delay"), referral and first appointment with TB services ("appointment delay"), first appointment and diagnosis ("diagnostic delay"), and diagnosis and treatment initiation ("treatment delay"). Total delay was defined as the period between date of symptom onset and the start of treatment.

Individual cohort review and ETS records were linked using the unique ETS identifier. People diagnosed post mortem were excluded from healthcare delay analyses. This analysis focused on passively identified cases who attended healthcare services after becoming unwell. People who self-referred to TB services, or were referred following active screening by PHE HPTs, as contacts of a TB case or by port health or occupational health were excluded. People referred from private providers $(n=5)$ were excluded from healthcare delay analysis as it was unclear if the main referrer was from primary or secondary care. In 
addition, unrealistic delay times that were probably entered in error (i.e. presentation delay/healthcare delay $<1$ day) were excluded.

\section{Analysis}

We described case characteristics stratified by cohort review-ETS linkage status. We then described completeness of presentation delay and healthcare delay outcome data by the case characteristics. We then described distributions of delay (expressed as median and interquartile range (IQR)) for all cases, and stratified by sputum smear status.

Potential risk factors were selected according to evidence from the literature [7-10], clinical plausibility in the England setting, and availability on ETS or cohort review databases. We used logistic regression to determine whether there was an association between linkage status and case characteristic to assess completeness of delay outcome data and whether outcome data completeness varied by case characteristic. We used random effects models to assist in controlling for unexplained heterogeneity between LADs across the South East (due to heterogeneous populations and settings). We allowed the model constant to vary by including $\mathrm{LAD}$ as a random effect if there was strong statistical evidence $(\mathrm{p}<0.05)$ of LAD heterogeneity. For the main analysis we used univariate and multivariable accelerated failure time models to model the time to event occurrence (delay time) as a continuous variable separately for presentation and healthcare delay outcomes. The regression parameter from accelerated failure time models can be expressed as a "time ratio" (TR): the ratio of the change in the time to the outcome event per unit increase of the independent variable. We used quantile-quantile (QQ) plots to assess agreement with accelerated failure time model assumptions that the fraction of cases with a risk factor yet to reach an end-point is proportional to the fraction without the risk factor (for categorised variables we compared each level with the base level). We selected the survival time distribution from the Weibull, exponential, loglog, lognormal and $\gamma$ distributions which minimised the Akaike's Information Criterion (AIC) score. We visually compared the observed survival distributions (Kaplan-Meier curves) against survival curves predicted by the final models to ensure reasonable fit to the data. We modelled LAD as a random effect, confirming the appropriateness of this, as described earlier. All risk factors for an outcome were considered as a potential confounder in multivariable analysis. Interactions between a priori defined potential effect modifiers were investigated by comparing models with and without the interaction term (excluding other variables) using a likelihood ratio test with a p-value of $<0.05$ determining evidence to retain the interaction term for multivariable analysis. As a sensitivity analysis, we deleted cases with the longest $1 \%$ of presentation and

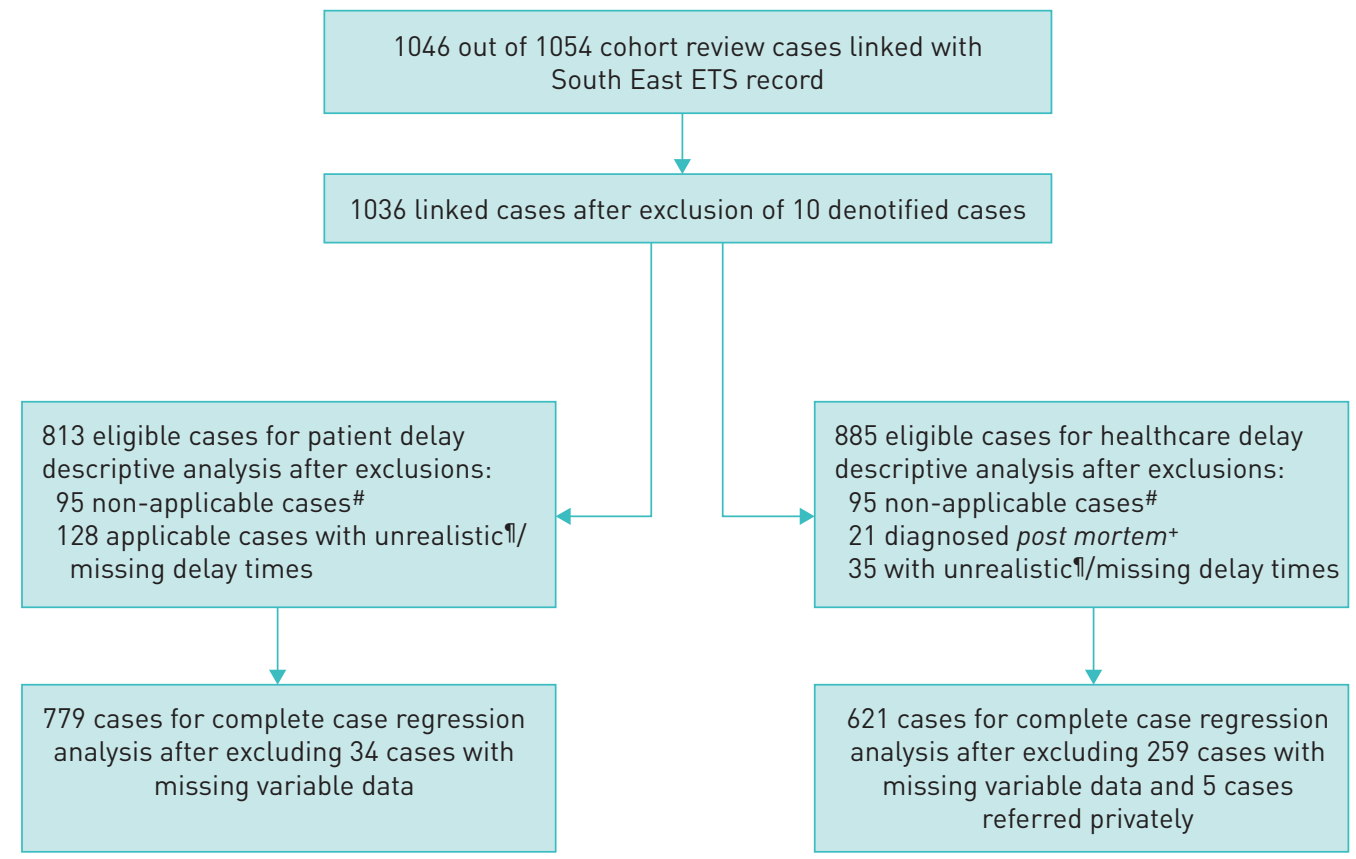

FIGURE 1 Case data flow. ETS: Enhanced Tuberculosis Surveillance system. ${ }^{\#}$ : identified as tuberculosis contacts/by port health/by occupational health; ${ }^{\text {I: }}$ delay of $<1$ day; ${ }^{+}$: these cases are included in descriptive analysis of "decision" and "referral" delays, but not other descriptive analyses, nor regression analyses of healthcare delay. 
TABLE 1 Presentation delay and healthcare delay by descriptive characteristics of people with pulmonary tuberculosis (TB), South East England, 2011-2015

\begin{tabular}{|c|c|c|c|c|}
\hline & \multicolumn{2}{|c|}{ Presentation delay } & \multicolumn{2}{|c|}{ Healthcare delay } \\
\hline & $\begin{array}{c}\text { Cases with delay } \\
\text { data }\end{array}$ & Delay days & $\begin{array}{c}\text { Cases with delay } \\
\text { data }\end{array}$ & Delay days \\
\hline All & 813 & $30(11-72)$ & 885 & $40(13-89)$ \\
\hline \multicolumn{5}{|l|}{ Year } \\
\hline 2011 & 38 & $51(10-111)$ & 44 & $34(8-99)$ \\
\hline 2012 & 102 & 30 (12-70) & 118 & 50 (14-99) \\
\hline 2013 & 224 & $28(9-60)$ & 232 & $32(14-72)$ \\
\hline 2014 & 232 & $29(12-62)$ & 257 & $44(14-94)$ \\
\hline 2015 & 217 & $31(12-91)$ & 234 & 43 (12-98) \\
\hline Unknown & 0 & & 0 & \\
\hline \multicolumn{5}{|l|}{ Health protection team } \\
\hline Hampshire Isle of Wight & 132 & $33(15-67)$ & 132 & 39 (15-83) \\
\hline Kent & 236 & $31(13-86)$ & 265 & $37(9-87)$ \\
\hline Surrey Sussex & 134 & $45(17-111)$ & 153 & $28(9-70)$ \\
\hline Thames Valley & 311 & $21(8-53)$ & 335 & $52(18-102)$ \\
\hline Unknown & 0 & & 0 & \\
\hline \multicolumn{5}{|l|}{ Cases by area TB incidence } \\
\hline$<20 / 100000$ & 554 & $31(13-83)$ & 615 & $43(14-95)$ \\
\hline$\geqslant 20 / 100000$ & 259 & $24(9-61)$ & 270 & $32(10-85)$ \\
\hline Unknown & 0 & & 0 & \\
\hline \multicolumn{5}{|l|}{ Rurality } \\
\hline Rural & 70 & $39(13-82)$ & 78 & $38(16-96)$ \\
\hline Urban & 743 & $29(11-70)$ & 807 & $41(12-89)$ \\
\hline Unknown & 0 & & 0 & \\
\hline \multicolumn{5}{|l|}{ Sex } \\
\hline Male & 485 & $30(12-81)$ & 528 & $35(10-78)$ \\
\hline Female & 327 & $30(11-62)$ & 356 & $48(19-111)$ \\
\hline Unknown & 1 & $1(1-1)$ & 1 & $327(327-327)$ \\
\hline \multicolumn{5}{|l|}{ Age group years } \\
\hline $0-14$ & 9 & $6(4-20)$ & 10 & $45(33-51)$ \\
\hline $15-44$ & 444 & $29(12-67)$ & 489 & $34(10-79)$ \\
\hline $45-64$ & 200 & $31(193-80)$ & 211 & $43(14-96)$ \\
\hline$\geqslant 65$ & 160 & $27(9-84)$ & 174 & $50(17-129)$ \\
\hline Unknown & 0 & & 0 & \\
\hline \multicolumn{5}{|l|}{ Time resident in UK years } \\
\hline$<2$ years & 96 & $33(15-68)$ & 105 & $31(10-62)$ \\
\hline $2-10$ years & 248 & $28(10-61)$ & 274 & $34(10-83)$ \\
\hline$\geqslant 11$ years & 167 & $23(9-61)$ & 174 & $55(19-116)$ \\
\hline UK-born & 283 & $31(10-92)$ & 309 & $44(16-99)$ \\
\hline Unknown & 19 & $64(23-121)$ & 23 & $26(5-56)$ \\
\hline \multicolumn{5}{|l|}{ Social risk factor } \\
\hline None & 704 & $28(10-66)$ & 768 & $43(14-98)$ \\
\hline 1 & 66 & $50(26-121)$ & 74 & $23(7-71)$ \\
\hline$\geqslant 2$ & 43 & 34 (17-88) & 43 & $18(5-65)$ \\
\hline Unknown & 0 & & 0 & \\
\hline \multicolumn{5}{|l|}{ QIMD } \\
\hline 1 (least) & 140 & $31(10-73)$ & 158 & $46(18-96)$ \\
\hline 2 & 139 & $29(13-61)$ & 156 & $47(16-114)$ \\
\hline 3 & 191 & $26(8-67)$ & 208 & $42(12-99)$ \\
\hline 4 & 231 & $31(13-72)$ & 247 & $40(12-85)$ \\
\hline 5 (most) & 112 & 34 (12-100) & 146 & $20(7-59)$ \\
\hline Unknown & 0 & & 0 & \\
\hline \multicolumn{5}{|l|}{ Ethnicity } \\
\hline Not white & 501 & $27(10-61)$ & 541 & $38(11-83)$ \\
\hline White & 312 & $35(13-92)$ & 342 & $42(15-100)$ \\
\hline Unknown & 0 & & 0 & \\
\hline
\end{tabular}




\section{TABLE 1 Continued}

\begin{tabular}{|c|c|c|c|c|}
\hline & \multicolumn{2}{|c|}{ Presentation delay } & \multicolumn{2}{|c|}{ Healthcare delay } \\
\hline & $\begin{array}{c}\text { Cases with delay } \\
\text { data }\end{array}$ & Delay days & $\begin{array}{c}\text { Cases with delay } \\
\text { data }\end{array}$ & Delay days \\
\hline \multicolumn{5}{|c|}{ Language barriers ${ }^{\#}$} \\
\hline Present & 88 & $32(15-96)$ & NC & \\
\hline Absent & 725 & $30(11-68)$ & NC & \\
\hline Unknown & 0 & & NC & \\
\hline \multicolumn{5}{|c|}{ Mental health comorbidity ${ }^{\#}$} \\
\hline Present & 31 & 43 (19-135) & NC & \\
\hline Absent & 782 & $30(11-68)$ & NC & \\
\hline Unknown & 0 & & NC & \\
\hline \multicolumn{5}{|l|}{$\begin{array}{l}\text { Physical health } \\
\text { comorbidity }\end{array}$} \\
\hline Present & 75 & $30(11-76)$ & 799 & $41(13-89)$ \\
\hline Absent & 738 & $31(12-56)$ & 86 & $33(15-95)$ \\
\hline Unknown & 0 & & 0 & \\
\hline \multicolumn{5}{|c|}{ Post mortem diagnosis } \\
\hline Yes & 8 & $8(5-91)$ & NC & \\
\hline No & 805 & $30(11-72)$ & NC & \\
\hline Unknown & 0 & & NC & \\
\hline \multicolumn{5}{|c|}{ Previous TB diagnosis } \\
\hline Yes & 51 & $31(10-92)$ & 57 & $38(16-70)$ \\
\hline No & 744 & $30(11-67)$ & 810 & $42(13-94)$ \\
\hline Unknown & 18 & $48(7-105)$ & 14 & $13(5-19)$ \\
\hline \multicolumn{5}{|l|}{ Sputum smear } \\
\hline Positive & 379 & $31(12-84)$ & 406 & $25(8-66)$ \\
\hline Negative & 300 & $27(11-65)$ & 330 & 51 (20-107) \\
\hline Unknown & 134 & $30(11-64)$ & 149 & 59 (27-132) \\
\hline \multicolumn{5}{|l|}{ Chest radiography } \\
\hline Abnormal: TB & NC & & 642 & $38(12-83)$ \\
\hline Not TB or NAD & NC & & 61 & 56 (19-129) \\
\hline Unknown & NC & & 182 & $43(15-88)$ \\
\hline \multicolumn{5}{|l|}{ Culture status } \\
\hline Positive & NC & & 720 & $38(12-79)$ \\
\hline Negative & NC & & 165 & 59 (19-132) \\
\hline Unknown & NC & & 0 & \\
\hline \multicolumn{5}{|l|}{ Referred by } \\
\hline Primary care & NC & & 340 & 51 (21-102) \\
\hline Secondary care & NC & & 420 & $27(8-78)$ \\
\hline Prison & NC & & 4 & $40(10-67)$ \\
\hline Private & NC & & 5 & 162 (29-179) \\
\hline Unknown & NC & & 116 & $54(20-99)$ \\
\hline
\end{tabular}

healthcare delays due to potentially weaker compliance with the assumptions of accelerated failure time models at outlying values, also providing a check as to the influence of outlying values.

Data were analysed in STATA 14 (STATACorp, College Station, TX, USA). Research ethics approval was not required as this study analysed routine data, with a primary aim to improve public health and clinical practice in the South East. Regulation 3 of the Health Service Regulations 2002 provided legal exemption from the Common Law Duty of Confidence, reflecting the intent to control a communicable disease [11].

\section{Results}

Figure 1 describes cases extracted from cohort review datasets and linked with ETS, then retained for analyses. Clinical and demographic characteristics of linked and unlinked cases were broadly similar (supplementary table S1). Presentation delay data were missing in $14 \%$ of cases otherwise eligible for analysis, and healthcare delay data in 4\% (supplementary table S2). Incomplete presentation delay data 
were associated with age $\geqslant 65$ years, smear negativity and not initiating treatment. Cases with and without healthcare delay data appeared to have similar characteristics. Median (IQR) total delay was 88 (49-163) days for all people with pTB, 79 (42-146) days in smear-positive cases and 95 (53-170) days in those with smear-negative disease. Table 1 describes the median presentation and healthcare delay stratified by case characteristics. Median presentation delay was slightly longer in people with smear-positive disease (31 days) than those with smear-negative (27 days) disease. Median healthcare delay was longer in smear-negative (51 days) than smear-positive (25 days) cases.

Breakdown of healthcare delay by smear status is shown in table 2. Decision delay accounted for the greatest part of healthcare delay. Median diagnostic delay was longer in those with smear-negative disease.

\section{Analytic epidemiology}

For both models, QQ plots excluded strong curvilinear relationships, but showed some moderate departures from a straight line, particularly at outlying values (supplementary figures S2 and S3). We selected the Weibull distribution to fit all models, because it had the lowest AIC (supplementary table S3). Comparison of single variable models and full multivariable models with and without the mixed effect suggested strong statistical evidence $(\mathrm{p}<0.05)$ that there was variation in delay between LAD areas. Therefore, this effect was incorporated by modelling LAD as a random effect. Inspection of the observed and predicted (from the full model) survival curves showed a close approximation (supplementary figures S4 and S5).

\section{Presentation delay}

Table 3 presents the single-variable and multivariable analysis results. On single-variable analysis there was no strong evidence of interaction between time in UK and social risk factor $(p=0.29)$, the only interaction considered. On multivariable analysis, longer presentation delay was associated with mental health barriers (TR 2.06, 95\% CI 1.22-3.50), and language barriers (TR 1.40, 95\% CI 1.01-1.94). Shorter presentation delay was associated with age $0-14$ years compared to cases aged $>64$ years (TR $0.23,95 \%$ CI $0.09-0.59$ ). The longer presentation delay associated with presence of a mental health comorbidity did not remain after exclusion of $1 \%$ outliers (adjusted TR 1.14, 95\% CI 0.69-1.86; supplementary table S4). Other associations remained largely unchanged.

\section{Healthcare delay}

On single-variable analysis, there was no evidence for an interaction between time in UK and sex $(\mathrm{p}=0.99)$. On multivariable analysis (table 4$)$, shorter healthcare delay was associated with smear positivity (TR 0.58, 95\% CI 0.47-0.70), male sex (TR 0.74, 95\% CI 0.60-0.91), having two or more social risk factors (TR 0.59, 95\% CI 0.38-0.94), secondary-care referral to TB services compared to primary-care referral (TR $0.63,95 \%$ CI $0.51-0.78$ ) and being in the UK for <2 years compared to UK-born cases (TR 0.47, 95\% CI $0.32-0.67)$. No significant difference was detected in healthcare delay for non-UK-born cases notified $\geqslant 11$ years after immigration compared to UK-born cases (TR 1.06, 95\% CI 0.76-1.47). After exclusion of the top $1 \%$ of outliers there was weaker evidence for a decreased healthcare delay in males (TR $0.86,95 \%$ CI 0.70-1.04; supplementary table S5). Other associations were robust to sensitivity analysis. Chest radiography was excluded from complete case analysis because $182(21 \%)$ out of 880 cases for healthcare delay analysis were missing chest radiography data, and there was no strong evidence of an association on multivariable analysis when added back into the final random-effects complete case multivariable analysis model (TR 0.76, 95\% CI 0.50-1.15; $\mathrm{p}=0.193 ; \mathrm{n}=518$ ). Its exclusion from this model did not alter the TR of the remaining variables by $\geqslant 20 \%$.

\section{TABLE 2 Median delay for different classifications of healthcare delay, South East of England, 2011-2015, and stratified by}

smear status

\begin{tabular}{|c|c|c|c|c|c|c|c|c|c|c|}
\hline & \multicolumn{2}{|c|}{ All healthcare delay ${ }^{\#}$} & \multicolumn{2}{|c|}{ Decision delay } & \multicolumn{2}{|c|}{ Referral delay } & \multicolumn{2}{|c|}{ Diagnostic delay ${ }^{\#}$} & \multicolumn{2}{|c|}{$\begin{array}{c}\text { Treatment initiation } \\
\text { delay }\end{array}$} \\
\hline & Cases & Delay days & Cases & Delay days & Cases & Delay days & Cases & Delay days & Cases & Delay days \\
\hline Any & 885 & $40(13-89)$ & 784 & $26(4-73)$ & 788 & $2(0-8)$ & 605 & $2(0-14)$ & 889 & $1(0-3)$ \\
\hline Negative & 330 & $51(20-107)$ & 286 & $27(5-79)$ & 282 & $3(0-13)$ & 241 & $8(1-31)$ & 330 & $1(0-4)$ \\
\hline Positive & 406 & $25(8-66)$ & 365 & $17(3-63)$ & 369 & $1(0-6)$ & 266 & $1(0-4)$ & 410 & $1(0-2)$ \\
\hline
\end{tabular}

Data are presented as $\mathrm{n}$ or median (interquartile range). All categories exclude cases known to have been referred by public health contact tracing or screening. ${ }^{\#}$ : excludes cases diagnosed post mortem. 


\begin{tabular}{|c|c|c|c|c|}
\hline & Crude time ratio $(95 \% \mathrm{CI})$ & p-value & Adjusted time ratio $(95 \% \mathrm{CI})$ & p-value \\
\hline \multicolumn{5}{|l|}{ Ethnicity } \\
\hline Not white & Ref. & & Ref. & \\
\hline White & $1.22(0.99-1.50)$ & 0.063 & $1.08(0.82-1.42)$ & 0.601 \\
\hline \multicolumn{5}{|c|}{ Mental health barriers } \\
\hline No & Ref. & & Ref. & \\
\hline Yes & $2.26(1.37-3.75)$ & 0.001 & $2.06(1.22-3.50)$ & 0.007 \\
\hline \multicolumn{5}{|l|}{ Rurality } \\
\hline Rural & Ref. & & Ref. & \\
\hline Urban & $0.92(0.65-1.32)$ & 0.656 & $0.88(0.61-1.27)$ & 0.500 \\
\hline \multicolumn{5}{|l|}{$\begin{array}{l}\text { Physical health } \\
\text { comorbidity }\end{array}$} \\
\hline No & Ref. & & Ref. & \\
\hline Yes & $0.86(0.62-1.20)$ & 0.366 & $0.75(0.54-1.05)$ & 0.091 \\
\hline \multicolumn{5}{|l|}{ Language barriers } \\
\hline No & Ref. & & Ref. & \\
\hline Yes & $1.25(0.92-1.71)$ & 0.160 & $1.40(1.01-1.94)$ & 0.042 \\
\hline \multicolumn{5}{|l|}{ Sex } \\
\hline Female & Ref. & & Ref. & \\
\hline Male & $1.10(0.90-1.34)$ & 0.335 & $1.04(0.85-1.27)$ & 0.698 \\
\hline \multicolumn{5}{|l|}{ Prior TB diagnosis } \\
\hline No & Ref. & & Ref. & \\
\hline Yes & $1.36(0.92-2.02)$ & 0.125 & $1.01(0.68-1.51)$ & 0.953 \\
\hline \multicolumn{5}{|l|}{ Age group years? } \\
\hline$\geqslant 65$ & Ref. & & Ref. & \\
\hline $45-64$ & $1.30(0.97-1.74)$ & 0.078 & $1.27(0.95-1.72)$ & 0.103 \\
\hline $15-44$ & $0.94(0.72-1.21)$ & 0.615 & $1.05(0.79-1.41)$ & 0.718 \\
\hline $0-14$ & $0.22(0.08-0.56)$ & 0.002 & $0.23(0.09-0.59)$ & 0.002 \\
\hline \multicolumn{5}{|l|}{$\begin{array}{l}\text { Social risk factors } \\
\text { present }^{+}\end{array}$} \\
\hline None & Ref. & & Ref. & \\
\hline 1 & $1.44(1.01-2.05)$ & 0.043 & $1.18(0.82-1.71)$ & 0.362 \\
\hline$\geqslant 2$ & $1.02(0.65-1.59)$ & 0.936 & $0.76(0.47-1.21)$ & 0.248 \\
\hline \multicolumn{5}{|l|}{ Time resident in UK ${ }^{\S}$} \\
\hline UK-born & Ref. & & Ref. & \\
\hline$\geqslant 11$ years & $1.01(0.77-1.18)$ & 0.947 & $0.99(0.71-1.39)$ & 0.959 \\
\hline $2-10$ years & $0.74(0.59-0.95)$ & 0.016 & $0.77(0.56-1.04)$ & 0.090 \\
\hline$<2$ years & $0.86(0.62-1.18)$ & 0.350 & $0.84(0.58-1.22)$ & 0.366 \\
\hline \multicolumn{5}{|l|}{ QIMD $f$} \\
\hline 1 (least deprived) & Ref. & & Ref. & \\
\hline 2 & $0.83(0.59-1.16)$ & 0.276 & $0.86(0.64-1.24)$ & 0.358 \\
\hline 3 & $0.93(0.58-1.28)$ & 0.668 & $0.98(0.73-1.36)$ & 0.878 \\
\hline 4 & $1.08(0.79-1.49)$ & 0.619 & $1.04(0.75-1.43)$ & 0.818 \\
\hline 5 (most deprived) & $1.19(0.81-1.75)$ & 0.381 & $1.20(0.86-1.85)$ & 0.341 \\
\hline \multicolumn{5}{|c|}{$\begin{array}{l}\text { QIMD: Quintile of Index of Multiple Deprivation. Bold type represents statistical significance at a p-value } \\
\leqslant 0.05 \text {. \#: all models include a random effect on } 62 \text { local authority districts; } 7 \text { : Wald } p \text {-value for overall } \\
\text { effect of age group categorical variable crude }<0.001 \text {, adjusted } 0.003 ;{ }^{+} \text {: Wald } p \text {-value for overall effect of } \\
\text { social risk factor categorical variable crude } 0.128 \text {, adjusted } 0.275 ;{ }^{\S} \text { : Wald } p \text {-value for overall effect of time } \\
\text { in UK categorical variable crude } 0.058 \text {, adjusted } 0.247 ;{ }^{*} \text { : Wald p-value for overall effect of QIMD } \\
\text { categorical variable crude } 0.306 \text {, adjusted } 0.474 \text {. }\end{array}$} \\
\hline
\end{tabular}

\section{Discussion}

Our cohort faced a median delay of 88 days from symptom onset to starting treatment, 11 days longer than the England median in 2016 [12]. For those with smear-negative disease, healthcare delay accounted for most of the delay, but presentation and healthcare delay were roughly equal for smear-positive cases. Making the decision to refer to TB specialists accounted for the majority of healthcare delay for all cases. Following referral, diagnosis usually occurred within 2 days, although diagnosis took approximately a week longer (and at least a month for 25\%) for smear-negative cases. Different risk factors were associated with presentation delay compared to healthcare delay. 


\begin{tabular}{|c|c|c|c|c|}
\hline & Crude time ratio $(95 \% \mathrm{CI})$ & p-value & Adjusted time ratio $(95 \% \mathrm{CI})$ & p-value \\
\hline \multicolumn{5}{|l|}{ Ethnicity } \\
\hline Not white & Ref. & & Ref. & \\
\hline White & $1.15(0.92-1.43)$ & 0.213 & $1.15(0.87-1.51)$ & 0.325 \\
\hline \multicolumn{5}{|l|}{$\begin{array}{l}\text { Physical health } \\
\text { comorbidity }\end{array}$} \\
\hline No & Ref. & & Ref. & \\
\hline Yes & $1.05(0.74-1.50)$ & 0.786 & $1.10(0.78-1.56)$ & 0.583 \\
\hline \multicolumn{5}{|l|}{ Sex } \\
\hline Female & Ref. & & Ref. & \\
\hline Male & $0.69(0.56-0.85)$ & $<0.001$ & $0.74(0.60-0.91)$ & 0.005 \\
\hline \multicolumn{5}{|c|}{ Prior TB diagnosis } \\
\hline No & Ref. & & Ref. & \\
\hline Yes & $0.92(0.62-1.37)$ & 0.690 & $0.95(0.65-1.41)$ & 0.815 \\
\hline \multicolumn{5}{|c|}{ Age group years? } \\
\hline$\geqslant 65$ & Ref. & & Ref. & \\
\hline $45-64$ & $0.81(0.59-1.12)$ & 0.207 & $0.78(0.57-1.09)$ & 0.144 \\
\hline $15-44$ & $0.68(0.51-0.90)$ & 0.008 & $0.79(0.57-1.09)$ & 0.151 \\
\hline $0-14$ & $0.45(0.13-1.58)$ & 0.212 & $0.55(0.17-1.85)$ & 0.337 \\
\hline \multicolumn{5}{|c|}{ Time resident in $\mathrm{UK}^{+}$} \\
\hline UK-born & Ref. & & Ref. & \\
\hline$\geqslant 11$ years & $1.08(0.81-1.44)$ & 0.600 & $1.06(0.76-1.47)$ & 0.741 \\
\hline $2-10$ years & $0.81(0.64-1.04)$ & 0.105 & $0.81(0.60-1.09)$ & 0.161 \\
\hline$<2$ years & $0.56(0.39-0.79)$ & 0.001 & $0.47(0.32-0.67)$ & $<0.001$ \\
\hline \multicolumn{5}{|c|}{ Area-level incidence } \\
\hline Low/medium & Ref. & & Ref. & \\
\hline High & $0.83(0.59-1.17)$ & 0.323 & $1.04(0.73-1.47)$ & 0.838 \\
\hline \multicolumn{5}{|l|}{ Referred by } \\
\hline Primary care & Ref. & & Ref. & \\
\hline Secondary & $0.65(0.53-0.81)$ & $<0.001$ & $0.63(0.51-0.78)$ & $<0.001$ \\
\hline Prison & $0.53(0.15-1.87)$ & 0.323 & $0.61(0.18-2.10)$ & 0.432 \\
\hline \multicolumn{5}{|l|}{ Sputum smear } \\
\hline Negative & Ref. & & Ref. & \\
\hline Positive & $0.60(0.49-0.74)$ & $<0.001$ & $0.58(0.47-0.70)$ & $<0.001$ \\
\hline \multicolumn{5}{|c|}{$\begin{array}{l}\text { Social risk factors } \\
\text { present }^{f}\end{array}$} \\
\hline None & Ref. & & Ref. & \\
\hline 1 & $0.71(0.50-1.00)$ & 0.05 & $0.91(0.64-1.28)$ & 0.578 \\
\hline$\geqslant 2$ & $0.54(0.34-0.86)$ & 0.009 & $0.59(0.38-0.94)$ & 0.025 \\
\hline \multicolumn{5}{|c|}{$\begin{array}{l}\text { Bold type represents statistical significance at a } p \text {-value } \leqslant 0.05 .^{*} \text { : all models include a random effect on } \\
62 \text { local authority districts; }{ }^{\text {ๆ }} \text { : Wald } p \text {-value for overall effect of age group categorical variable crude } 0.039 \text {, } \\
\text { adjusted } 0.40 ;{ }^{+} \text {: Wald } p \text {-value for overall effect of time in UK categorical variable crude } 0.002 \text {, adjusted } \\
<0.001 ;{ }^{\S} \text { : Wald } p \text {-value for overall effect of referred by categorical variable crude }<0.001 \text {, adjusted }<0.001 \text {; } \\
f: \text { Wald p-value for overall effect of social risk factor categorical variable crude } 0.007 \text {, adjusted } 0.08 \text {. }\end{array}$} \\
\hline
\end{tabular}

Language barriers were associated with a $40 \%$ longer time to presentation; although known to be associated with poorer TB treatment outcome [13], our findings suggest an important role in accessing care, which we believe was not previously described in the literature. Our simple classification means we cannot assess linguistic ability, nor have we assessed knowledge and attitudes to TB. Therefore, non-language barriers to presentation such as stigma, TB knowledge and healthcare eligibility issues [7] may confound this relationship. Mental health barriers were associated with a doubling of presentation delay on complete case analysis, which would be consistent with the literature suggesting that mental health disorders (specifically depression) can delay healthcare seeking [14]. However, mental health barriers were not associated with presentation delay on sensitivity analysis removing the $1 \%$ of outlying (longest) delays, suggesting that our estimates for mental health barriers were overly influenced by cases with outlying delays. The possibility of model misspecification for these cases makes any association between mental health barriers and presentation delay difficult to quantify, and will need to be confirmed on further research. 
Smear negativity was associated with a $42 \%$ longer healthcare delay, and longer delays associated with smear status has been recorded in other studies $[7,10]$. Delays are probably due to diagnostic challenges of a negative first-line investigation, but also because positive smear is associated with more severe and clinically and radiologically obvious disease [15-17]. Consistent with previous observational data from the UK or United States examining healthcare delay or total delay, we found longer delays in females $[8,18$, 19], native-born cases [9, 10, 18-20], cases with longer time since migration [19] and cases referred by general practitioners [10]. One explanation for the above populations experiencing longer delays would be a low index of clinical suspicion in the examining clinician for cases whom they perceive as being at low risk of TB. This could result in delayed investigation and/or referral to specialist services, and would be consistent with our finding of the intervals between presentation and eventual referral to specialist $\mathrm{TB}$ services accounting for the large majority of healthcare delay. Alternatively, from our data we cannot be certain that some of the apparent delay in referral could also be due to administrative rather than clinical factors, but it seems less likely that this would be differential for the factors identified. Primary care may be an exception; differential access of the examining clinician to first-line investigations and specialist advice, as well as potentially a lower index of suspicion to consider onward referral to TB specialists are likely to contribute to longer delays compared to secondary care.

Older age has been associated with longer total delay $[9,10,19]$, increased delays being most pronounced when comparing adults with children [9, 19], and thought to be due to difficulties in recognising TB symptoms among other comorbidities, or less typical TB presentation. Similarly, we observed shorter presentation delay in children, and weak evidence of trends to longer healthcare delay with older age; we may have detected a significant effect with a larger cohort giving greater statistical power. In people with multiple social risk factors (but not a single social risk factor), we found weak evidence of shorter presentation delay, and strong evidence of shorter healthcare delay. Shorter total delay has been observed in groups with social risk factors by researchers in the UK [18] and USA [20], but not consistently by others $[8,19]$. Shorter delays for those with multiple social risk factors could be due to case finding and specific referral pathways in what is likely to be a high-risk group, and greater awareness of increased risk by clinicians, but effects may be specific to presentation delay/healthcare delay and differ by region.

Data completeness may have resulted in selection bias and loss of power, particularly for healthcare delay where case attrition was greatest. The recall bias inherent in determining dates of clinical events (particularly symptom onset which was dependent on case recall) leave these data vulnerable to misclassification, probably non-differential. The referrer to TB services variable was used as a proxy for the type of clinician/agency to whom the case first presented. This may have resulted in differential misclassification as cases are probably more likely to self-present to secondary care after failure to be diagnosed in primary care rather than vice versa, thus underestimating the healthcare delay in primary care. There was an increasing departure from linearity on QQ plots at the most extreme values (typically the top 3-10\%) of delays. As few cases took this long to present/commence treatment there is likely to be increased variability, so the departure from linearity at extreme values is not unexpected. Inspection of the fitted compared to observed values for both models was somewhat reassuring. However, we cannot rule out that the model results will not be affected by more severe deviations from the accelerated failure time assumptions.

Our findings support continued initiatives to enable access to care for migrant populations to minimise presentation delays, which would have wider benefits for potentially marginalised communities. Delays in referral to specialist services, probably in large part due to low suspicion of TB as a possible diagnosis, but also diagnostic complexity of smear-negative cases, were responsible for significant healthcare delays. This may be further compounded for people with extrapulmonary sites of disease. Shorter delays to diagnosis have been noted in London compared to the lowest incidence areas of England, including the South East [19]; our findings are most generalisable to low-incidence settings with relatively low ethnic diversity, and similar referral pathways. Improving awareness of $\mathrm{TB}$ in such populations remains a significant challenge. TB is becoming less common in England [3], therefore clinicians will become less likely to encounter cases regularly, potentially further lowering their clinical suspicion. Recognising the local epidemiology, and that an increasing proportion of cases are now either UK-born or longer-term migrants [3], and not in groups traditionally perceived as "high-risk", may help. Therefore, multifaceted approaches to increase clinician awareness of TB risk groups and clinical presentations, to implement systems enabling early case recognition, to maximise the yield from sputum smear investigations and to ensure the rapid diagnosis of smear negative cases are required to address the delays in diagnosis and achieve further TB control.

Conflict of interest: None declared. 


\section{References}

1 Rieder H. Epidemiologic Basis of Tuberculosis Control. Paris, International Union Against Tuberculosis and Lung Disease, 1999; pp. 11-17.

2 National Institute for Health and Care Excellence (NICE). NICE Guideline NG33: Tuberculosis. London, NICE, 2016.

3 Public Health England. Tuberculosis in England 2018 Report (Presenting Data to End of 2017). London, Public Health England, 2018.

4 Public Health England. Tuberculosis in England 2016 Report (Presenting Data to End of 2015). London, Public Health England, 2016.

5 Public Health England. Collaborative Tuberculosis Strategy for England 2015 to 2020. London, Public Health England, 2015. Contract No.: 2014596.

6 Anderson C, White J, Abubakar I, et al. Raising standards in UK TB control: introducing cohort review. Thorax 2014; 69: 187-189.

7 Storla DG, Yimer S, Bjune GA. A systematic review of delay in the diagnosis and treatment of tuberculosis. BMC Public Health 2008; 8: 15.

8 Sultan H, Haroon S, Syed N. Delay and completion of tuberculosis treatment: a cross-sectional study in the West Midlands, UK. I Public Health 2013; 35: 12-20.

9 Saldana L, Abid M, McCarthy N, et al. Factors affecting delay in initiation of treatment of tuberculosis in the Thames Valley, UK. Public Health 2013; 127: 171-177.

10 Paynter S, Hayward A, Wilkinson P, et al. Patient and health service delays in initiating treatment for patients with pulmonary tuberculosis: retrospective cohort study. Int J Tuberc Lung Dis 2004; 8: 180-185.

11 Taylor MJ. Legal bases for disclosing confidential patient information for public health: distinguishing between health protection and health improvement. Med Law Rev 2015; 23: 348-374.

12 Public Health England. Tuberculosis in England 2017 Report (Presenting Data to End of 2016). London, Public Health England, 2017.

13 Gardam M, Verma G, Campbell A, et al. Impact of the patient-provider relationship on the survival of foreign born outpatients with tuberculosis. J Immigr Minor Health 2009; 11: 437-445.

14 Sweetland AC, Kritski A, Oquendo MA, et al. Addressing the tuberculosis-depression syndemic to end the tuberculosis epidemic. Int J Tuberc Lung Dis 2017; 21: 852-861.

15 Otero L, Ugaz R, Dieltiens G, et al. Duration of cough, TB suspects' characteristics and service factors determine the yield of smear microscopy. Trop Med Int Health 2010; 15: 1475-1480.

16 El-Sony A, Enarson D, Khamis A, et al. Relation of grading of sputum smears with clinical features of tuberculosis patients in routine practice in Sudan. Int J Tuberc Lung Dis 2002; 6: 91-97.

17 Ralph AP, Ardian M, Wiguna A, et al. A simple, valid, numerical score for grading chest $\mathrm{x}$-ray severity in adult smear-positive pulmonary tuberculosis. Thorax 2010; 65: 863-869.

18 Gissing S, Bindra R. Diagnostic delay in tuberculosis in Yorkshire \& Humber, UK: 2013-16 data. Eur Respir J 2018; 52: Suppl. 62, PA5267.

19 Loutet MG, Sinclair C, Whitehead N, et al. Delay from symptom onset to treatment start among tuberculosis patients in England, 2012-2015. Epidemiol Infect 2018; 146: 1511-1518.

20 Wallace RM, Kammerer JS, Iademarco MF, et al. Increasing proportions of advanced pulmonary tuberculosis reported in the United States: are delays in diagnosis on the rise? Am J Respir Crit Care Med 2009; 180 $1016-1022$. 